\title{
Mutation Site Dependent Variability of Cardiac Events in Japanese LQT2 Form of Congenital Long-QT Syndrome
}

\author{
Iori Nagaoka, MD; Wataru Shimizu, MD*; Hideki Itoh, MD; Satoshi Yamamoto, MD; \\ Tomoko Sakaguchi, MD; Yuko Oka, MD; Keiko Tsuji, MS; Takashi Ashihara, MD; \\ Makoto Ito, MD; Hidetada Yoshida, MD**; Seiko Ohno, MD**; \\ Takeru Makiyama, MD**; Yoshihiro Miyamoto, MD ${ }^{\dagger}$; Takashi Noda, MD*; \\ Shiro Kamakura, MD*; Masaharu Akao, MD**; Minoru Horie, MD
}

\begin{abstract}
Background In the LQT2 form of long QT syndrome (LQTS), mutation sites are reported to correlate with clinical phenotypes in Caucasians, but the relationship in Asian patients remains unknown. The present study was designed to determine whether the location of $\mathrm{KCNH} 2$ mutations would influence the arrhythmic risk in LQT2 patients.

Methods and Results In 118 genetically-confirmed LQT2 patients (69 families, $62 \mathrm{KCNH} 2$ mutations), the ECG parameters, Schwartz scores, and the incidence of cardiac events, defined as syncope, aborted cardiac arrest, and sudden cardiac death, were evaluated. To examine the effect of mutation sites, the participants were divided accordingly: pore $(n=56)$ and non-pore $(n=62)$ groups. The corrected QTend interval was significantly greater in the pore than in the non-pore group (QTc; $522 \pm 63 \mathrm{~ms}$ vs $490 \pm 49 \mathrm{~ms}, \mathrm{p}=0.002)$. In this study, the clinical course of each of the probands did not differ according to the mutation sites, whereas non-probands carrying the pore site mutation experienced their first cardiac events at significantly younger age than those with the non-pore site mutation (log-rank, $\mathrm{p}=0.0005)$.

Conclusions In a Japanese LQT2 cohort, family members with the pore site mutation were at higher arrhythmic risk than those with the non-pore site mutation. (Circ J 2008; 72: 694-699)
\end{abstract}

Key Words: Arrhythmia; Long-QT syndrome; QTc interval; Risk factors; Torsade de pointes

$\mathbf{T}$ he long QT syndrome (LQTS) is an inherited arrhythmogenic disease of the structurally normal heart that may cause sudden death. LQTS is characterized by an abnormality in myocardial repolarization that leads to prolongation of the QT interval, morphological changes in $\mathrm{T}$ waves and torsades-de-pointes (Tdp) type of ventricular tachycardia on surface ECGs!,2 To date, 8 distinct genes responsible for LQTS have been identified, including those of Andersen (LQT7) and Timothy (LQT8) syndromes: on chromosome 11q15.5 (KCNQ1; LQT1), 7q35-36 (KCNH2; LQT2), 3p21 (SCN5A; LQT3), 4q25-27 (ANKB; LQT4), 21q22 (KCNE1; LQT5), 21q22 (KCNE2; LQT6), 17q23 (KCNJ2; LQT7) and 12p13.3 (CACN1C; LQT8) $)^{3-10}$

Moss et al ${ }^{11}$ extensively examined the relationships between the site of mutation and clinical phenotype in approxi-

(Received October 17, 2007; revised manuscript received December 11, 2007; accepted December 13, 2007)

Department of Cardiovascular and Respiratory Medicine, Shiga University of Medical Science, Otsu, *Division of Cardiology, Department of Internal Medicine, National Cardiovascular Center, Suita, **Department of Cardiovascular Medicine, Kyoto University Graduate School of Medicine, Kyoto and Laboratory of Molecular Genetics, National Cardiovascular Center, Suita, Japan

Mailing address: Minoru Horie, MD, Department of Cardiovascular and Respiratory Medicine, Shiga University of Medical Science, Seta Tsukinowa-cho, Otsu 520-2192, Japan. E-mail: horie@belle. shiga-med.ac.jp

All rights are reserved to the Japanese Circulation Society. For permissions, please e-mail: cj@j-circ.or.jp mately 44 different LQT2-related $K C N H 2$ mutations. They reported that subjects with causative mutations in the pore region ( $\mathrm{n}=38$, amino acid residues 550 through 650$)$ had more severe clinical manifestations and experienced a higher frequency ( $74 \%$ vs $35 \%$; $\mathrm{p}<0.001)$ of arrhythmia-related cardiac events occurring at younger age than did subjects with non-pore mutations $(\mathrm{n}=166)$.

In LQT1, based on the United States portion of the International LQTS Registry ( $\mathrm{n}=425)$, the Netherlands' LQTS Registry ( $\mathrm{n}=93)$, and the Japanese LQTS Registry $(\mathrm{n}=82)$, 600 patients with $K C N Q 1$ mutations were classified into 2 groups of patients with transmembrane and C-terminus mutations and their clinical phenotypes were examined!2 That study found that patients with transmembrane mutations were at increasing risk for cardiac events (hazard ratio, 2.06; $\mathrm{p}<0.001)$. Shimizu et al also studied the mutation sitedependent differences in 95 LQT1 patients from a multicenter Japanese population and also found that patients with transmembrane mutations were at higher risk of cardiac events and had longer QTc and Tpeak-end intervals! ${ }^{13}$

In Japanese LQT2 patients, mutation site dependency is unclear, although this has been reported in Caucasian patients. Therefore, in the present study we aimed to compare the genotype and phenotype relationship, according to the classification adopted by Moss et al, ${ }^{11}$ in 118 Japanese LQT2 patients who were genetically identified in the 3 genetic centers in Japan and had no other mutations in LQTSrelated genes (except LQT4 and 8). 
Table 1 KCNH2 Mutations by Location, Amino-Acid Coding, Type of Mutation, and Reported Functional Effects

\begin{tabular}{|c|c|c|c|c|c|c|}
\hline & $\begin{array}{l}\text { No. of } \\
\text { families }\end{array}$ & $\begin{array}{l}\text { No. of } \\
\text { subjects }\end{array}$ & Position & Exon & Type of mutation & $\begin{array}{c}\text { Functional effect in } \\
\text { expression studies }\end{array}$ \\
\hline \multicolumn{7}{|l|}{ Pore regions } \\
\hline$A 561 T$ & 1 & 1 & S5 & 7 & Missense & Trafficking defect (22) \\
\hline$A 561 \mathrm{~V}$ & 1 & 1 & S5 & 7 & Missense & Dominant negative (23) \\
\hline$W 563 C^{*}$ & 1 & 1 & Pore & 7 & Missense & \\
\hline$W 563 G^{*}$ & 1 & 2 & Pore & 7 & Missense & \\
\hline$C 566 F^{*}$ & 1 & 1 & Pore & 7 & Missense & \\
\hline$G 572 S$ & 2 & 4 & Pore & 7 & Missense & \\
\hline$M 574 V^{*}$ & 1 & 3 & Pore & 7 & Missense & \\
\hline$R 582 L$ & 1 & 2 & Pore & 7 & Missense & \\
\hline$R 582 C$ & 1 & 1 & Pore & 7 & Missense & \\
\hline$G 584 C^{*}$ & 1 & 2 & Pore & 7 & Missense & \\
\hline G590V* & 1 & 3 & Pore & 7 & Missense & \\
\hline $1593 \mathrm{~V}^{*}$ & 1 & 1 & Pore & 7 & missense & \\
\hline$K 595 N^{*}$ & 1 & 2 & Pore & 7 & Missense & \\
\hline$K 595 E^{*}$ & 1 & 1 & Pore & 7 & Missense & \\
\hline G601S & 2 & 5 & Pore & 7 & Missense & Trafficking defect $(22,24)$ \\
\hline G604S & 2 & 2 & Pore & 7 & Missense & \\
\hline$S 606 P^{*}$ & 1 & 1 & Pore & 7 & Missense & \\
\hline T613M & 2 & 3 & Pore & 7 & Missense & Dominant negative (25) \\
\hline A614V & 4 & 6 & Pore & 7 & Missense & Dominant negative (26) \\
\hline T623I & 1 & 1 & Pore & 7 & Missense & Trafficking defect (22) \\
\hline G628S & 1 & 2 & Pore & 7 & Missense & Trafficking defect (22) \\
\hline N629K & 1 & 1 & Pore & 7 & Missense & Dominant negative (27) \\
\hline N633S & 1 & 1 & Pore & 7 & Missense & \\
\hline K638del & 1 & 1 & S6 & 7 & Deletion & \\
\hline F640del* & 1 & 1 & S6 & 7 & Deletion & \\
\hline$S 641 F$ & 1 & 3 & S6 & 7 & Missense & \\
\hline$V 644 F$ & 1 & 4 & S6 & 7 & Missense & \\
\hline Subtotal & 34 & 56 & & & & \\
\hline \multicolumn{7}{|l|}{ Non-pore regions } \\
\hline \multicolumn{7}{|l|}{$N$-terminal regions } \\
\hline$V 41 A^{*}$ & 1 & 1 & $N$-term & 2 & Missense & \\
\hline$Y 43 D^{*}$ & 1 & 3 & $N$-term & 2 & Missense & \\
\hline$E 50 f s+10 X^{*}$ & 1 & 1 & $N$-term & 2 & Deletion/frameshift & \\
\hline$G 53 S^{*}$ & 1 & 1 & $N$-term & 2 & Missense & \\
\hline 82-84insIAQ & 1 & 1 & $N$-term & 2 & Insertion & \\
\hline$F 106 L^{*}$ & 1 & 1 & $N$-term & 3 & Missense & \\
\hline$D 111 V^{*}$ & 1 & 1 & $N$-term & 3 & Missense & \\
\hline$V 115 M^{*}$ & 1 & 1 & $N$-term & 3 & Missense & \\
\hline$P 151 f s+179 X$ & 1 & 1 & $N$-term & 3 & Insertion/frameshift & \\
\hline G187-A190del* & 1 & 3 & $N$-term & 4 & Deletion & \\
\hline R312-S318del* & 1 & 2 & $N$-term & 5 & Deletion & \\
\hline S320L & 1 & 1 & $N$-term & 5 & Missense & \\
\hline P334L & 1 & 1 & $N$-term & 5 & Missense & \\
\hline$K 364 f s+3 X^{*}$ & 1 & 3 & $N$-term & 5 & Insertion/deletion/frameshift & \\
\hline$K 386 f s+3 X^{*}$ & 1 & 4 & $N$-term & 5 & Insertion/frameshift & \\
\hline \multicolumn{7}{|c|}{ Transmembrane domains other than pore regions } \\
\hline$Q 391 X$ & 1 & 2 & S1 & 6 & Nonsense & \\
\hline$\widetilde{F} 471 f s+50 X^{*}$ & 1 & 1 & $S 1-S 2$ & 6 & Deletion/frameshift & \\
\hline$I 489 F^{*}$ & 1 & 1 & $S 1-S 2$ & 6 & Missense & \\
\hline A490T & 1 & 1 & $S 1-S 2$ & 6 & Missense & Current density $\downarrow$ (28) \\
\hline$H 492 Y^{*}$ & 1 & 2 & $S 1-S 2$ & 6 & Missense & \\
\hline$W 497 X^{*}$ & 1 & 3 & S3 & 6 & Nonsense & \\
\hline$D 501 N$ & 1 & 1 & S3 & 6 & Missense & \\
\hline$R 534 C$ & 1 & 2 & S4 & 7 & Missense & Trafficking defect (22) \\
\hline \multicolumn{7}{|l|}{ C-terminal region } \\
\hline$Q 738 X^{*}$ & 1 & 2 & C-term & 9 & Nonsense & \\
\hline G745-G749del, Fins/fs + 56X* & 1 & 1 & $C$-term & 9 & Insertion/deletion/frameshift & \\
\hline$R 752 \mathrm{~W}$ & 1 & 2 & C-term & 9 & Missense & Trafficking defect (22) \\
\hline$S 818 L$ & 1 & 1 & C-term & 10 & Missense & Reduced Ikr current (29) \\
\hline$P 846 T^{*}$ & 1 & 1 & C-term & 10 & Missense & \\
\hline$W 853 f s+14 X^{*}$ & 1 & 2 & $C$-term & 10 & Deletion/frameshift & \\
\hline$R 863 X$ & 1 & 2 & C-term & 10 & Nonsense & \\
\hline$L 911 f s+6 X^{*}$ & 1 & 3 & $C$-term & 12 & Deletion/frameshift & \\
\hline$R 912 f s+63 X^{*}$ & 1 & 2 & C-term & 12 & Insertion/frameshift & \\
\hline S1029fs $+23 X^{*}$ & 1 & 3 & $C$-term & 13 & Deletion/frameshift & \\
\hline$P 1034 f s+23 X^{*}$ & 1 & 3 & $C$-term & 13 & Insertion/frameshift & \\
\hline$A 1144 T^{*}$ & 1 & 2 & $C$-term & 15 & Missense & \\
\hline Subtotal & 35 & 62 & & & & \\
\hline
\end{tabular}

*Novel mutation.

del, deletion; ins, insertion; fs, first amino acid affected by a frameshift (number after fs is number of amino acids before termination); term, terminus. 
Table 2 Clinical Characteristics of Pore and Non-Pore Mutations

\begin{tabular}{|c|c|c|c|}
\hline & Pore $(n=56)$ & Non-pore $(n=62)$ & $p$ value \\
\hline \multicolumn{4}{|l|}{ Demographics } \\
\hline Female gender (\%) & $33(59 \%)$ & $42(68 \%)$ & 0.344 \\
\hline Proband (\%) & $33(59 \%)$ & $34(55 \%)$ & 0.712 \\
\hline Age (years) at baseline ECG (range) & $31 \pm 18(7-74)$ & $31 \pm 16(2-71)$ & 0.920 \\
\hline Age (years) at first event (range) & $16 \pm 10(5-48)$ & $20 \pm 13(5-71)$ & 0.203 \\
\hline \multicolumn{4}{|l|}{ Diagnosis } \\
\hline Schwartz score & $5.3 \pm 1.6$ & $4.5 \pm 1.8$ & 0.017 \\
\hline Schwartz score $\geq 4$ (\%) & $47(84 \%)$ & $41(66 \%)$ & 0.034 \\
\hline \multicolumn{4}{|l|}{ ECG measurements } \\
\hline Heart rate (beats/min) & $65 \pm 13$ & $64 \pm 15$ & 0.537 \\
\hline$R R(m s)$ & $953 \pm 188$ & $975 \pm 186$ & 0.510 \\
\hline$Q T_{\text {end }}(m s)$ & $505 \pm 79$ & $482 \pm 69$ & 0.089 \\
\hline$Q T_{\text {peak }}(\mathrm{ms})$ & $377 \pm 67$ & $382 \pm 65$ & 0.650 \\
\hline$T_{p e a k-e n d}(\mathrm{~ms})$ & $129 \pm 55$ & $99 \pm 41$ & 0.001 \\
\hline Corrected $Q T_{\text {end }}(\mathrm{ms})$ & $522 \pm 63$ & $490 \pm 49$ & 0.002 \\
\hline Corrected $Q T_{\text {peak }}(\mathrm{ms})$ & $389 \pm 62$ & $388 \pm 47$ & 0.927 \\
\hline Corrected Tpeak-end (ms) & $134 \pm 52$ & $101 \pm 42$ & $<0.001$ \\
\hline Torsade de pointes (\%) & $17(30 \%)$ & $18(29 \%)$ & 1.000 \\
\hline T-wave alternans (\%) & $7(13 \%)$ & $4(7 \%)$ & 0.346 \\
\hline Notched T wave (\%) & $43(77 \%)$ & $32(52 \%)$ & 0.007 \\
\hline \multicolumn{4}{|l|}{ Cardiac events } \\
\hline All cardiac events $(\%)$ & $38(68 \%)$ & $32(52 \%)$ & 0.092 \\
\hline Syncope (\%) & $36(64 \%)$ & $32(52 \%)$ & 0.194 \\
\hline Aborted cardiac arrest/SCD (\%) & $6(11 \%)$ & $2(3 \%)$ & 0.145 \\
\hline \multicolumn{4}{|l|}{ Therapy } \\
\hline$\beta$-blocker therapy & $26(53 \%)$ & $21(36 \%)$ & 0.117 \\
\hline Pacemaker $(\%)$ & $1(2 \%)$ & 0 & 1.000 \\
\hline Sympathectomy (\%) & 0 & 0 & 1.000 \\
\hline Defibrillator $(\%)$ & $1(2 \%)$ & $2(3 \%)$ & 1.000 \\
\hline
\end{tabular}

Data are mean value $\pm S D$ or number $(\%)$ of subjects.

$E C G$, electrocardiography; $S C D$, sudden cardiac death.

\section{Methods}

\section{Study Population}

The study population consisted of 118 patients from 69 unrelated Japanese LQT2 families enrolled from 3 institutes in Japan: National Cardiovascular Center, Kyoto University Graduate School of Medicine and Shiga University of Medical Science. The $\mathrm{KCNH} 2$ mutations were confirmed in all patients by using standard genetic tests ${ }^{14-17}$ Screening for mutations in KCNQ1, SCN5A, KCNE1, KCNE2, and $K C N J 2$ was also conducted, and patients with compound mutations of $\mathrm{KCNH} 2$ and/or additional mutations in these LQTS-related genes were excluded from the analysis. Symptomatic patients were defined as $\mathrm{KCNH} 2$ mutation carriers who experienced at least 1 episode of syncope (ie, complete loss of consciousness, or cardiac arrest requiring cardiac resuscitation), while asymptomatic patients were those without these events. Follow-up was censored at age 50 years to avoid the influence of coronary artery disease on cardiac events.

\section{Genetic Analysis and Characterization}

Genomic DNA was isolated from venous blood by use of the QIAamp DNA blood midikit (Qiagen, Hilden, Germany). The protocol for genetic analysis was approved by the institutional ethics committee and was performed under its guidelines. Established primer settings were used to amplify the entire coding regions of the known LQTS genes from genomic DNA $!^{4-17}$ Denaturing high-performance liquid chromatography (DHPLC) was used for screening. For aberrant conformers, direct sequencing techniques were performed as described elsewhere ${ }^{3}$ PCR products were denatured at $95^{\circ} \mathrm{C}$ for $5 \mathrm{~min}$ then analyzed by DHPLC. PCR fragments presenting abnormal signals in the DHPLC analysis were subsequently sequenced by the dideoxynucleotide chain termination method with fluorescent dideoxynucleotides in an ABI 3130 genetic analyzer (PE Applied Biosystems).

The pore region of the $\mathrm{KCNH} 2$ channel was defined as the area extending from S5 to the mid-portion of S6 involving amino acid residues 550 through 650 , according to a previous report!1 The non-pore region included the N-terminus region, transmembrane domains other than the pore region and the $\mathrm{C}$-terminus region.

\section{Clinical Characterization}

Routine demographic data and basal 12-lead ECGs were obtained for all subjects at the time of enrollment in each institute and there was at least yearly follow-up contact. All ECGs were taken before or without $\beta$-blocker medication. The ECG parameters measured from the basal recordings were the RR, QTend, QTpeak and $\mathrm{T}_{\text {peak-end }}(\mathrm{QT}$ end-QTpeak) intervals. The latter is thought to reflect the transmural dispersion of ventricular repolarization (TDR) ${ }^{18-20}$ The ratedependent QT intervals were corrected for heart rate by Bazett's method? ${ }^{21}$ The QT peak was defined as the time interval between QRS onset and the peak of the positive $\mathrm{T}$ wave or the nadir of the negative $\mathrm{T}$ wave. $\mathrm{T}_{\text {peak-end was then } \mathrm{ob}-}$ tained by calculating QTend minus QTpeak.

These parameters were measured manually in lead V5 averaged from 2 or 3 consecutive beats. Bifid T waves other than $U$ waves were included in the QT measurements. If ECG recordings were obtained during a cardiac event, the patients were requested to undergo the examination again after improving. Measurements were performed by 3 investigators who were completely unaware of the patient's clinical and genetic status. There were no significant differences in the measured data between the investigators, and the 
mean values were used for analysis. LQTS-related cardiac events were defined as syncope, aborted cardiac arrest, or unexpected sudden death.

\section{Statistical Analyses}

All data are expressed as the mean value \pm SD. The Student's t-test was used to compare continuous data between mutations located in the pore region and those in the non-pore region. Differences in frequencies were analyzed by the chi-square test. Time to the first cardiac event (syncope, cardiac arrest, or sudden cardiac death) before initiation of $\beta$-blocker therapy and before age 50 years was determined by Kaplan-Meier cumulative estimates. Two-sided probability values $<0.05$ were considered statistically significant. Statistical calculations were performed with SPSS software (version 11.01J, Chicago, IL, USA).

\section{Results}

\section{Genetic Characteristics}

Table 1 lists the $K C N H 2$ mutations we identified, classified by location, number of patients with these causative mutations, coding effects (missense, insertion, deletion and frameshift) and functional outcomes. We identified 62 different $K C N H 2$ mutations among the 69 LQTS families: 42 missense, 16 deletion/insertion, 11 frameshift and 4 nonsense mutations. There were $27(44 \%)$ mutations causing amino acid changes in the pore region and $35(56 \%)$ mutations within the non-pore regions (15 in the N-terminus, 8 in the non-pore transmembrane, and 12 in the C-terminus). In the pore mutations there were $25(93 \%)$ missense mutations and the remaining 2 were protein deletions (K638del and F640del).

In contrast, the non-pore mutations included more significantly complex mutations such as deletion, insertion, frameshift or nonsense mutations that resulted in truncation of channel proteins $(15 / 35,43 \%)$. Thirty-five mutations $(56 \%$, 11 in the pore region and 24 in the non-pore regions) were novel and indicated by asterisk in Table 1 . Functional effects by cellular electrophysiologic tests have been reported in only 12 of the 62 mutations (19\%);22-29 however, all those previous reports indicated that the $\mathrm{KCNH} 2$ mutations had loss-of-function effects and made the IKr current reduce or disappear. Four pore mutations had dominant-negative effects, 4 pore mutations and 2 non-pore mutations had trafficking defects, and 2 non-pore mutations reduced the IKr current.

\section{Clinical Characteristics}

Table 2 is a comparison of the clinical characteristics of the 56 patients with pore mutations and the 62 patients with non-pore mutations. There were no significant differences between the 2 groups regarding gender, the percentage of probands and the age at baseline ECG recording. Diagnostic LQTS scores of Schwartz et $\mathrm{al}^{30}$ were noticeably greater in the pore group. RR and QT peak intervals were comparable; however, corrected QTend and Tpeak-end intervals were much longer in the pore than in the non-pore group. Although the incidence of TdP and T-wave alternans did not differ between groups, notched $\mathrm{T}$ waves were more frequently seen in the pore group ( $\mathrm{p}=0.007$ vs non-pore group). The incidence of cardiac events and the introduction of $\beta$-blocker therapy were not statistically different between the 2 groups.
A

Total $(\mathrm{N}=118)$

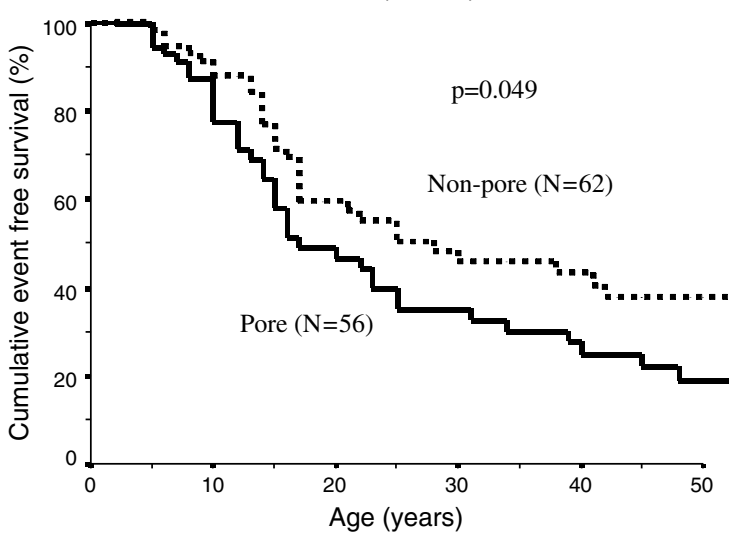

B

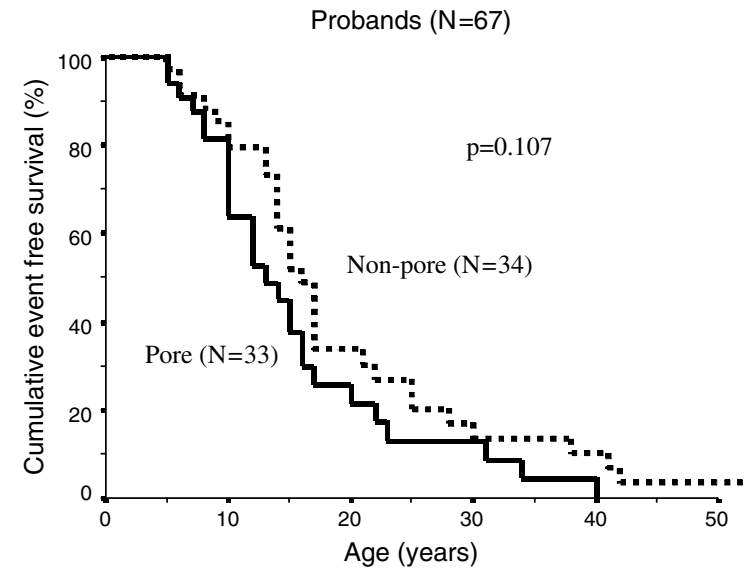

C

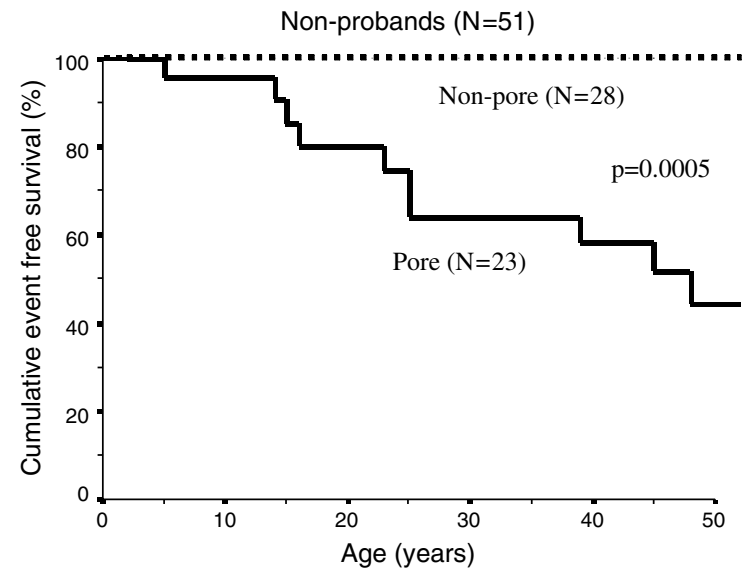

Fig 1. (A) Kaplan-Meier cumulative cardiac event-free survival curves from birth through to age 50 years for the total of 118 patients with $\mathrm{KCNH} 2$ mutations located in the pore $(\mathrm{n}=56$, smooth line) and non-pore $(n=62$, dotted line) regions. The pore group patients experienced their first cardiac event at a younger age than the non-pore group (log-rank, $\mathrm{p}=0.049$ ). The difference was caused mainly by the high firstevent rate in non-probands. Kaplan-Meier cumulative cardiac event-free survival curves for 67 probands (B) and 51 non-probands (C) with pore mutations (smooth line) and non-pore mutations (dotted line).

\section{Clinical Course by Mutation Location}

Fig 1A shows the Kaplan-Meier cumulative cardiac event-free survival curves from birth through to age 50 years for 118 patients (pore group, $n=56$; non-pore group, $n=62$ ). The pore-group patients experienced their first cardiac event at a younger age than the non-pore group $(\log$-rank, $\mathrm{p}=$ 
Table 3 Clinical Characteristics of Pore and Non-Pore Mutations in Non-Probands

\begin{tabular}{|c|c|c|c|}
\hline & Pore $(n=23)$ & Non-pore $(n=28)$ & $p$ value \\
\hline \multicolumn{4}{|l|}{ Demographics } \\
\hline Female gender $(\%)$ & $14(61 \%)$ & $19(68 \%)$ & 0.769 \\
\hline Age (years) at baseline ECG (range) & $42 \pm 20(9-74)$ & $33 \pm 20(2-71)$ & 0.124 \\
\hline \multicolumn{4}{|l|}{ Diagnosis } \\
\hline Schwartz score & $4.7 \pm 1.5$ & $3.5 \pm 1.7$ & 0.008 \\
\hline Schwartz score $\geq 4(\%)$ & $18(78 \%)$ & $12(43 \%)$ & 0.021 \\
\hline \multicolumn{4}{|l|}{ ECG measurements } \\
\hline Heart rate (beats/min) & $65 \pm 15$ & $70 \pm 17$ & 0.251 \\
\hline$R R(m s)$ & $959 \pm 179$ & $894 \pm 179$ & 0.201 \\
\hline$Q T_{\text {end }}(\mathrm{ms})$ & $480 \pm 51$ & $441 \pm 54$ & 0.0011 \\
\hline$Q T_{\text {peak }}(\mathrm{ms})$ & $352 \pm 47$ & $352 \pm 53$ & 0.974 \\
\hline$T_{\text {peak-end }}(\mathrm{ms})$ & $128 \pm 46$ & $89 \pm 30$ & 0.001 \\
\hline Corrected QTend (ms) & $494 \pm 45$ & $470 \pm 40$ & 0.044 \\
\hline Corrected $Q T_{\text {peak }}(\mathrm{ms})$ & $364 \pm 49$ & $374 \pm 40$ & 0.423 \\
\hline Corrected Tpeak-end (ms) & $131 \pm 43$ & $96 \pm 32$ & 0.002 \\
\hline Torsade de pointes (\%) & $1(4 \%)$ & 0 & 0.451 \\
\hline T-wave alternans (\%) & 0 & 0 & - \\
\hline Notched T wave (\%) & $17(74 \%)$ & $14(50 \%)$ & 0.095 \\
\hline \multicolumn{4}{|l|}{ Cardiac events } \\
\hline All cardiac events $(\%)$ & $11(48 \%)$ & 0 & $<0.001$ \\
\hline Syncope $(\%)$ & $10(43 \%)$ & 0 & $<0.001$ \\
\hline Aborted cardiac arrest/SCD (\%) & $1(4 \%)$ & 0 & 0.451 \\
\hline \multicolumn{4}{|l|}{ Therapy } \\
\hline$\beta$-blocker therapy & $6(26 \%)$ & 0 & 0.006 \\
\hline Pacemaker (\%) & 0 & 0 & - \\
\hline Sympathectomy (\%) & 0 & 0 & - \\
\hline Defibrillator (\%) & 0 & 0 & - \\
\hline
\end{tabular}

Data are mean value $\pm S D$ or number (\%) of subjects.

Abbreviations see in Table 2.

0.049). We examined the clinical course of the 67 probands and 51 non-probands separately (Figs 1B,C). The clinical courses of the probands were not significantly different according to mutation site (Fig 1B), whereas in the non-pore group 28 non-probands remained asymptomatic and more than half had suffered from cardiac events by the age of 50 (Fig 1C). Therefore, the difference stemmed from markedly distinct prognoses among the non-probands.

Table 3 summarizes the clinical characteristics of the 51 non-probands. The absolute and corrected QTend and $T_{\text {peak-end }}$ intervals were all significantly greater in the pore than in the non-pore group. In the non-probands, the incidence of all cardiac events, syncope, and $\beta$-blocker therapy were significantly greater in the pore group than in the nonpore group.

\section{Discussion}

This study demonstrates that the clinical features of 118 Japanese LQT2 patients who had 62 different $K C N H 2$ mutations correlated with the mutation sites, but only in nonprobands. In probands, there was no significant relationship between mutation site and prognosis. Moss et al ${ }^{11}$ reported approximately 179 LQT2 patients based on 44 different KCNH2 mutations and those patients with pore mutations had significantly $(\mathrm{p}<0.0001)$ higher frequency of LQTSrelated cardiac events and longer QTc intervals than those with non-pore mutations. In contrast to their results, in the present study the mutation-dependent difference in prognosis was relatively small, though significant $(\mathrm{p}=0.049)$, when analyzed in the total patient cohort (Fig 1A). Indeed, the beneficial outcome of the non-pore patients stemmed from their family members (Fig 1C), and probands showed virtually similar prognosis to that of pore mutation carriers. Although Moss et al did not report separate sub-analysis of probands and family members, the percentage of family members in their non-pore group was significantly larger than that of the pore group ( $84 \%$ vs $57 \%, \mathrm{p}<0.001$ ). The very good prognosis of the non-pore mutation group in their study may have reflected that large number of family members.

The character of the mutation per se may be important as another reason for the variance between these 2 studies, as both had a similar number of LQT2 patients. Compared with the study by Moss et all ${ }^{11}$ the type of mutation in the present study was quite different: in our non-pore group, there were significantly more complex mutations, such as nonsense or frameshift, that caused the truncation of channel proteins $(15 / 35,43 \%)$ than in the report of Moss et al $(4 / 30,13 \%)$. For example, nonsense-mediated mRNA decay (NMD) has recently been reported to play an important role in reducing dominant negative suppression effects? Premature termination codon caused by either a deletion or insertion mutation would also cause NMD and thereby attenuate the severity of cardiac phenotypes. This different nature of the mutations may cause the apparently different prognosis of the non-pore mutation groups in each study.

In our pore site mutation group, there were only 2 inframe deletions, but no frameshift mutations (Table 1). Although it was practically very difficult to conduct every functional assay for each novel $\mathrm{KCNH} 2$ mutation identified here, some cellular electrophysiological effects are available in a small number of $K C N H 2$ mutations we found (Table 1). Several missense mutations in the pore region (such as A561V and T613M) have been shown to produce dominant negative suppression effects, a greater functional change predisposing to arrhythmic events. In contrast, functional assay of several missense mutations in the non-pore regions has revealed relatively smaller loss-of-function effects (such as with A490T or S818L) Greater functional disruption may also be reflected in the different prognosis 
of family members in the pore and non-pore groups (Fig 1).

Previously we reported that LQT1 patients with $K C N Q 1$ mutations located in the transmembrane regions, including the pore region, are at a higher risk of congenital LQTS-related cardiac events and longer QTc and Tpeak-end intervals than are patients with C-terminal mutations $!^{3}$ In LQT2, we have also demonstrated that $\mathrm{T}_{\text {peak-end, representing trans- }}$ mural dispersion of ventricular repolarization, ${ }^{19}$ is longer in pore patients than in non-pore patients (Table 2), supporting the finding that family members with pore mutations are more likely to suffer from LQTS-related cardiac events than those with a non-pore mutation.

\section{Study Limitations}

Cardiac events are not simply linked to the site of mutation in probands; there are other triggering factors such as modifier genes, including single nucleotide polymorphisms, 32 hypokalemia and bradycardia, which play significant roles in aggravating the symptoms of $\mathrm{KCNH} 2$ mutation carriers. The influence of these factors could be interpreted in the similar occurrence of cardiac events in the probands irrespective of mutation site, because the presence of symptoms usually caused the patient to agree to undergo genetic testing.

Regarding each mutation, the number of study patients was relatively small (at most 5), and the location of the mutations was scattered, even in the same pore region. The coding effect was also so various that we had limited ability to show arrhythmic risk according to a specific mutation site. Our cohort contained 35 novel $\mathrm{KCNH} 2$ mutations, and their functional outcomes were not available. Moreover, our study population included only Japanese, so more subjects per mutation and a greater spectrum of $\mathrm{KCNH} 2$ mutations in a worldwide study are needed to evaluate the arrhythmic risks associated with these mutations.

\section{Acknowledgments}

Dr W. Shimizu was supported by the Uehara Memorial Foundation, and with $\mathrm{Dr}$ M. Horie by grants from Ministry of Education, Culture, Sports, Science and Technology Leading Project for Biosimulation, and health sciences research grants (H18-Research on Human Genome-002) from the Ministry of Health, Labour and Welfare, Japan.

\section{References}

1. Schwartz PJ, Periti M, Malliani A. The long QT syndrome. Am Heart J 1975; 89: 378-390.

2. Moss AJ, Schwartz PJ, Crampton RS, Tzivoni D, Locati EH, MacCluer J, et al. The long-QT syndrome: Prospective longitudinal study of 328 families. Circulation 1991; 84: 1136-1144.

3. Keating M, Atkinson D, Dunn C, Timothy K, Vincent GM, Leppert $\mathrm{M}$. Linkage of a cardiac arrhythmia, the long QT syndrome, and the Harvey ras-1 gene. Science 1991; 252: 704-706.

4. Jiang C, Atkinson D, Towbin JA, Splawski I, Lehmann MH, Li H, et al. Two long QT syndrome loci map to chromosome 3 and 7 with evidence for further heterogeneity. Nat Genet 1994; 8: 141 - 147.

5. Schott J, Charpentier F, Peltier S, Foley P, Drouin E, Bouhour JB, et al. Mapping of a gene for long QT syndrome to chromosome 4q2527. Am J Hum Genet 1995; 57: 1114-1122.

6. Splawski I, Tristani-Firouzi M, Lehmann MH, Sanguinetti MC, Keating MT. Mutations in the hminK gene cause long QT syndrome and suppress IKs function. Nat Genet 1997; 17: $338-340$.

7. Abbott GW, Sesti F, Splawski I, Buck ME, Lehmann MH, Timothy $\mathrm{KW}$, et al. MiRP1 forms IKr potassium channels with HERG and is associated with cardiac arrhythmia. Cell 1999; 97: 175-187.

8. Keating MT, Sanguinetti MC. Molecular and cellular mechanisms of cardiac arrhythmias. Cell 2001; 104: 569-580.

9. Plaster NM, Tawil R, Tristani-Firouzi M, Canun S, Bendahhou S, Tsunoda A, et al. Mutations in Kir2.1 cause the developmental and episodic electrical phenotypes of Andersen's syndrome. Cell 2001; 105: $511-519$.

10. Splawski I, Timothy KW, Sharpe LM, Decher N, Kumar P, Bloise R, et al. $\mathrm{Ca}(\mathrm{V}) 1.2$ calcium channel dysfunction causes a multisystem disorder including arrhythmia and autism. Cell 2004; 119: 19-31.

11. Moss AJ, Zareba W, Kaufman ES, Gartman E, Peterson DR, Benhorin J, et al. Increased risk of arrhythmic events in long-QT syndrome with mutations in the pore region of the human ether-a-go-gorelated gene potassium channel. Circulation 2002; 105: 794-799.

12. Moss AJ, Shimizu W, Wilde AA, Towbin JA, Zareba W, Robinson JL, et al. Clinical aspects of type-1 long-QT syndrome by location, coding type, and biophysical function of mutations involving the KCNQ1 gene. Circulation 2007; 115: 2481-2489.

13. Shimizu W, Horie M, Ohno S, Takenaka K, Yamaguchi M, Shimizu $\mathrm{M}$, et al. Mutation site-specific differences in arrhythmic risk and sensitivity to sympathetic stimulation in the LQT1 form of congenital long QT syndrome. J Am Coll Cardiol 2004; 44: 117-125.

14. Splawski I, Shen J, Timothy KW, Vincent GM, Lehmann MH, Keating MT. Genomic structure of three long QT syndrome genes: KVLQT1, HERG, and KCNE1. Genomics 1998; 51: 86-97.

15. Wang Q, Li Z, Shen J, Keating MT. Genomic organization of the human SCN5A gene encoding the cardiac sodium channel. Genomics 1996; 34: 9-16.

16. Abbott GW, Sesti F, Splawski I, Buck ME, Lehmann MH, Timothy $\mathrm{KW}$, et al. MiRP1 forms $I_{K r}$ potassium channels with HERG and is associated with cardiac arrhythmia. Cell 1999; 97: 175-187.

17. Tristani-Firouzi M, Jensen JL, Donaldson MR, Sansone V, Meola G, Hahn A, et al. Functional and clinical characterization of KCNJ2 mutations associated with LQT7 (Andersen syndrome). J Clin Invest 2002; 110: $381-388$.

18. Shimizu W, Antzelevitch C. Sodium channel block with mexiletine is effective in reducing dispersion of repolarization and preventing torsade de pointes in LQT2 and LQT3 models of the long QT syndrome. Circulation 1997; 96: 2038-2047.

19. Yan GY, Antzelevitch C. Cellular basis for the normal $T$ wave and the electrocardiographic manifestations of the long-QT syndrome. Circulation 1998; 98: 1928-1936.

20. Shimizu W, Antzelevitch C. Cellular basis for the ECG features of the LQT1 form of the long-QT syndrome: Effects of $\beta$-adrenergic agonists and antagonists and sodium channel blockers on transmural dispersion of repolarization and torsade de pointes. Circulation 1998; 98: 2314-2322.

21. Bazett HC. An analysis of the time-relations of electrocardiograms. Heart 1920; 7: 353-370.

22. Anderson CL, Delisle BP, Anson BD, Kilby JA, Will ML, Tester DJ, et al. Most LQT2 mutations reduce kv11.1 (hERG) current by a class 2 (trafficking-deficient) mechanism. Circulation 2006; 113: 365-373.

23. Kagan A, Yu Z, Fishman GI, McDonald TV. The dominant negative LQT2 mutation A561V reduces wild-type HERG expression. J Biol Chem 2000; 275: $11241-11248$

24. Furutani M, Trudeau MC, Hagiwara N, Seki A, Gong Q, Zhou Z, et al. Novel mechanism associated with an inherited cardiac arrhythmia defective protein trafficking by the mutant HERG (G601S) potassium channel. Circulation 1999; 99: 2290-2294.

25. Huang FD, Chen J, Lin M, Keating MT, Sanguinetti MC. Long-QT syndrome-associated missense mutations in the pore helix of the HERG potassium channel. Circulation 2001; 104: 1071-1075.

26. Nakajima T, Furukawa T, Tanaka T, Katayama Y, Nagai R, Nakamura Y, et al. Novel mechanism of HERG current suppression in LQT2: Shift in voltage dependence of HERG inactivation. Circ Res 1998; 83: 415-422.

27. Yoshida H, Horie M, Otani H, Takano M, Tsuji K, Kubota T, et al. Characterization of a novel missense mutation in the pore of HERG in a patient with long QT syndrome. J Cardiovasc Electrophysiol 1999; 10: $1262-1270$.

28. Yoshida H, Horie M, Otani H, Kawashima T, Onishi Y, Sasayama S. Bradycardia-induced long QT syndrome caused by a de novo missense mutation in the S2-S3 inner loop of HERG. Am J Med Genet 2001; 98: $348-352$.

29. Nakajima T, Kurabayashi M, Ohyama Y, Kaneko Y, Furukawa T, Itoh $\mathrm{T}$, et al. Characterization of S818L mutation in HERG C-terminus in LQT2 modification of activation-deactivation gating properties. FEBS Lett 2000; 481: 197-203.

30. Schwartz PJ, Moss AJ, Vincent GM, Crampton RS. Diagnostic criteria for the long QT syndrome an update. Circulation 1993; 88: 782-784.

31. Gong Q, Zhang L, Vincent GM, Horne BD, Zhou Z. Nonsense mutations in hERG cause a decrease in mutant mRNA transcripts by nonsense-mediated mRNA decay in human long-QT syndrome. Circulation. 2007; 116: 17-24.

32. Ozawa T, Ito M, Tamaki S, Yao T, Ashihara T, Kita Y, et al. Gender and age effects on ventricular repolarization abnormality in Japanese general carriers of a G643S common single nucleotide polymorphism for the KCNQ1 gene. Circ J 2006; 70: 645-650. 